03

\title{
Термализация фотонов в микрорезонаторе
}

\author{
() Е.Д. Трифонов \\ Российский государственный педагогический университет им. А.И. Герцена, \\ 191186 Санкт-Петербург, Россия \\ e-mail: thphys@herzen.spb.ru
}

Поступила в редакцию 30.08.2017 г.

Предложено теоретическое объяснение образования спектральной конденсации фотонов в микрорезонаторе, основанное на решении кинетических уравнений, описывающих термализацию фотонного газа в результате комбинационного рассеяния на термостате атомарного газа.

DOI: 10.21883/OS.2018.01.45362.191-17

\section{Введение}

Для объяснения равновесного теплового излучения Эйнштейн предложил использовать стационарное решение кинетического уравнения для среднего числа фотонов, которое описывает поглощение, вынужденное и спонтанное излучения при переходах между определенными квантовыми состояниями термостата [1]. Чтобы распространить процесс установления термодинамического равновесия на более широкий интервал частот, потребовалось включить механизм рассеяния фотонов, что на феноменологическом уровне еще до появления квантовой механики было выполнено в работах Паули [2], Эйнштейна и Эренфеста [3]. С помощью квантовой механики такое рассмотрение было осуществлено в работах Компанейца [4], Зельдовича и Левича [5], исследовавших решения кинетических уравнений, описывающих рассеяние света на электронном газе.

Другим выдающимся достижением Эйнштейна в области квантовой статистической физики для идеального газа тождественных частиц с ненулевой массой покоя было теоретическое предсказание эффекта бозеэйнштейновской конденсации (БЭК): при достаточно низкой температуре „некоторое число молекул переходит в первое квантовое состояние (с нулевой кинетической энергией)“ [6]. Прямое экспериментальное подтверждение БЭК в идеальном газе было осуществлено лишь сравнительно недавно [7,8].

В последнее время появился интерес к исследованиям „конденсации фотонного газа““, в какой-то степени аналогичной БЭК. Эксперименты [9-11], демонстрирующие БЭК фотонов, предполагают термализацию состояния электромагнитного поля в микрорезонаторе при дополнительной лазерной накачке, поддерживающей среднее число фотонов в ограниченном спектральном интервале. Было обнаружено, что даже при комнатной температуре это приводит к заметной конденсации фотонов на нижней границе некоторого спектрального интервала.

В настоящей работе мы предлагаем теоретическое описание процесса спектральной конденсации фотонов с помощью решения кинетических уравнений, описывающих установление термодинамического равновесия в микрорезонаторе при рассеянии излучения на разреженном газе, играющем роль термостата.

\section{Рассеяние фотонов на термостате}

Пусть резонатор заполнен разреженным газом в состоянии термодинамического равновесия. Сначала рассмотрим рассеяние фотонов на одном атоме. Пусть возбуждение атома происходит под действием поля квантового радиационного осциллятора с волновым вектором k, а последующий переход атома в основное состояние и появление рассеянного излучения объясняется действием поля радиационного осциллятора с волновым вектором $\mathbf{k}^{\prime}$.

Атом будем рассматривать как двухуровневую электронную систему с электронными волновыми функциями $\varphi_{a}$ и $\varphi_{b}$ основного и возбужденного состояний с собственными значениями энергий $E_{a}$ и $E_{b}$. Для возбужденного электронного состояния мы будем учитывать радиационную ширину уровня энергии $Г / 2$. Кроме двух электронных состояний мы введем еще волновые функции поступательного движения атома (волны де Бройля)

$$
\psi_{\mathbf{p}}(\mathbf{r}, t)=\frac{1}{\sqrt{V}} \exp \left[i\left(\mathbf{p r}-\varepsilon_{\mathbf{p}} t\right)\right]
$$

где $\mathbf{r}-$ радиус-вектор положения атома, $\mathbf{p}-$ волновой вектор атома, $\varepsilon_{\mathbf{p}}=\hbar p^{2} / 2 M-$ частота, обусловленная кинетической энергией атома, имеющего массу $M, V-$ объем системы. Таким образом, базис одноатомных состояний будет иметь вид

$$
\begin{aligned}
& \psi_{a, \mathbf{p}}=\varphi_{a} \psi_{\mathbf{p}}, \\
& \psi_{b, \mathbf{p}^{\prime}}=\varphi_{b} \psi_{\mathbf{p}^{\prime}}
\end{aligned}
$$

Будем рассматривать взаимодействие этого атома с квантованным электромагнитным полем. В приближении Вигнера-Вайцхопфа [12] вероятность в единицу времени акта рассеяния, при котором происходит поглощение фотона с волновым вектором $\mathbf{k}$ и испускание 
фотона с волновым вектором $\mathbf{k}^{\prime}$, может быть представлена в виде $[13,14]$

$$
\begin{aligned}
W_{\mathbf{k}, \mathbf{k}^{\prime}}= & \frac{\pi d^{4} \omega \omega^{\prime}}{8 V^{2} \hbar^{2}} n\left(n^{\prime}+1\right) \frac{1}{\left(\omega_{b a}-\omega\right)^{2}+\Gamma^{2} / 4} \\
& \times \delta\left(\omega-\omega^{\prime}+\varepsilon_{\mathbf{p}}-\varepsilon_{\mathbf{p}+\mathbf{k}-\mathbf{k}^{\prime}}\right) .
\end{aligned}
$$

Здесь $d-$ модуль матричного элемента дипольного момента перехода, по направлению которого проведено усреднение, $n$ и $n^{\prime}-$ числа фотонов с волновыми векторами $\mathbf{k}$ и $\mathbf{k}^{\prime}$ в начальном состоянии электромагнитного поля. Дельта-функция обеспечивает закон сохранения энергии и описывает сдвиг частоты рассеянного света на величину, соответствующую изменению кинетической энергии атома в акте рассеяния.

Рассмотрим теперь рассеяние фотонов на идеальном газе таких атомов, который находится в состоянии термодинамического равновесия при температуре $T$. Для этого проведем суммирование вероятности (3) по ансамблю атомов, подчиняющемуся максвелловскому распределению

$$
N\left(2 \pi M k_{B} T / \hbar^{2}\right)^{-\frac{3}{2}} \exp \left(-\frac{\hbar^{2}\left(p_{x}^{2}+p_{y}^{2}+p_{z}^{2}\right)}{2 M k_{B} T}\right),
$$

где $N$ - полное число атомов в рассматриваемом объеме.

Аргумент $\delta$-функции в (3)

$$
\frac{\hbar p^{2}}{2 M}+c k-\frac{\hbar\left(\mathbf{p}+\mathbf{k}-\mathbf{k}^{\prime}\right)^{2}}{2 M}-c k^{\prime},
$$

выражающий закон сохранения энергии, может быть представлен в виде

$$
\frac{\hbar\left(\mathbf{p}, \mathbf{k}^{\prime}-\mathbf{k}\right)}{M}-c\left(k^{\prime}-k\right)-\frac{\hbar\left(\mathbf{k}^{\prime}-\mathbf{k}\right)^{2}}{2 M} .
$$

Выберем ось $Z$ в направлении вектора $\mathbf{k}^{\prime}-\mathbf{k}$. Тогда нашу $\delta$-функцию можно записать как

$$
\delta\left(\frac{\hbar p_{z} \xi}{M}-c\left(k^{\prime}-k\right)-\frac{\hbar \xi^{2}}{2 M}\right)=\frac{M}{\hbar \xi} \delta\left(p_{z}-\frac{M c\left(k^{\prime}-k\right)}{\hbar \xi}-\frac{\xi}{2}\right)
$$

где $\xi-$ модуль вектора $\xi=\mathbf{k}^{\prime}-\mathbf{k}$. После интегрирования по составляющим импульса атома вместо $\delta$-функции получим дополнительный множитель

$$
N \sqrt{\frac{M}{2 \pi k_{B} T \xi^{2}}} \exp \left(-\frac{1}{2 k_{B} T}\left(\frac{\Delta^{2} M}{\xi^{2}}+\hbar \Delta+\frac{\hbar^{2} \xi^{2}}{4 M}\right)\right),
$$

где $\Delta=c\left(k^{\prime}-k\right)$ - изменение частоты фотона.

Таким образом, вероятность рассеяния $n, n^{\prime} \rightarrow n-1$, $n^{\prime}+1$ в единицу времени на термостате может быть представлена как

$$
\begin{aligned}
& W_{\mathbf{k}, \mathbf{k}^{\prime}}^{T}=\frac{\pi d^{4} \omega \omega^{\prime}}{8 V^{2} \hbar^{2}} n\left(n^{\prime}+1\right) \frac{1}{\left(\omega_{b a}-\omega\right)^{2}+\Gamma^{2} / 4} \\
& \quad \times N \sqrt{\frac{M}{2 \pi k_{B} T \xi^{2}}} \exp \left(-\frac{1}{2 k_{B} T}\left(\frac{\Delta^{2} M}{\xi^{2}}+\hbar \Delta+\frac{\hbar^{2} \xi^{2}}{4 M}\right)\right),
\end{aligned}
$$

Содержащаяся в этом выражении экспонента $\exp \left(-\frac{\hbar \Delta}{2 k_{B} T}\right)$ отражает принцип детального равновесия, хотя также следует обратить внимание на его нарушение, обусловленное присутствием лоренциана. Отметим, что так как $\mathbf{k}^{\prime} \neq \mathbf{k}$, то $\xi \neq 0$. Экспонента

$$
\exp \left(-\frac{1}{2 k_{B} T} \frac{\Delta^{2} M}{\zeta^{2}}\right)
$$

ограничивает вероятность рассеяния с большим изменением $\Delta$ частоты фотона.

\section{Кинетические уравнения}

Процесс установления термодинамического равновесия фотонного газа на ограниченном спектральном интервале $\left(\omega_{\text {cutoff }}, \infty\right)$ резонатора будем описывать системой кинетических уравнений ${ }^{1}$

$$
\dot{n}_{\mathbf{k}}=\sum_{\mathbf{k}^{\prime} \neq \mathbf{k}}-W_{\mathbf{k}, \mathbf{k}^{\prime}}^{T}+W_{\mathbf{k}^{\prime}, \mathbf{k}}^{T},
$$

или, в упрощенных обозначениях,

$$
\dot{n}_{k}=\sum_{k \neq k^{\prime}}-n_{k}\left(n_{k^{\prime}}+1\right) W_{k k^{\prime}}+n_{k^{\prime}}\left(n_{k}+1\right) W_{k^{\prime}, k} .
$$

Здесь индексы $k$ и $k^{\prime}$ нумеруют различные радиационные осцилляторы, $n_{k}\left(n_{k^{\prime}}+1\right) W_{k k^{\prime}}-$ вероятности переходов в единицу времени при взаимодействии с термостатом, описывающих виртуальное поглощение фотона $k$-го осциллятора и виртуальное испускание фотона $j$-го осциллятора.

Так как каждое слагаемое в системе (12) встречается дважды: один раз с „плюсом“, другой раз - с „минусом“", то

$$
\sum_{k} \dot{n}_{k}=0
$$

и, следовательно, обеспечен закон сохранения среднего числа фотонов. Покажем, что если бы принцип детального равновесия выполнялся точно,

$$
e^{-\hbar \omega_{k} / k_{B} T} W_{k, k^{\prime}}=e^{-\hbar \omega_{k^{\prime}} / k_{B} T} W_{k^{\prime}, k} \equiv w_{k^{\prime}, k}
$$

\footnotetext{
${ }^{1}$ Кинетические уравнения, описывающие лазерное охлаждение газа частиц с ненулевой массой покоя и образование БЭК, рассматривались нами в $[13-15]$. О канонических распределениях для ансамблей с конечным числом частиц см. также [16].
} 
то стационарное решение системы (12) имело бы вид

$$
n_{k}=\frac{1}{\exp \frac{\hbar \omega_{k}-\mu}{k_{B} T}-1} .
$$

Действительно, каждое слагаемое в правой части уравнения (12) можно представить в виде

$$
-n_{k} n_{k^{\prime}}\left(1+\frac{1}{n_{k^{\prime}}}\right) W_{k k^{\prime}}+n_{k} n_{k^{\prime}}\left(1+\frac{1}{n_{k}}\right) W_{k^{\prime}, k},
$$

и поэтому в силу (15) и (16) имеем

$$
\begin{aligned}
\sum_{k} \dot{n}_{k}= & \sum_{k, k^{\prime} \neq k}-n_{k} n_{k^{\prime}} \exp \left(\frac{\hbar \omega_{k^{\prime}}-\mu}{k_{B} T}\right) W_{k k^{\prime}} \\
& +n_{k} n_{k^{\prime}} \exp \left(\frac{\hbar \omega_{k}-\mu}{k_{B} T}\right) W_{k^{\prime}, k}=0 .
\end{aligned}
$$

Это означает, что при установившемся термодинамическом равновесии фотоны образуют большой канонический ансамбль. Отметим, что полученный таким образом результат (17) не зависит от величин вероятностей $W_{k k^{\prime}}$ : достаточно лишь выполнение соотношения (16).

Величина химического потенциала $\mu$ может быть определена из условия сохранения среднего числа фотонов. Если при заданной температуре термостата полное число фотонов $n$ больше планковского, т. е. $n>n_{P}$, где

$$
n_{P}=\sum_{k} \frac{1}{\exp \frac{\hbar \omega_{k}}{k_{B} T}-1}
$$

то химический потенциал должен быть положительным, но меньше нижней границы $\omega_{\text {cutoff }}$ спектрального интервала, $0<\mu<\hbar \omega_{\text {cutoff. }}$ В этом случае распределение числа фотонов с течением времени будет приближаться к каноническому с образованием конденсата вблизи $\omega_{\text {cutoff }}$.

Таким образом, соотношение $n=n_{P}(T)$ можно рассматривать как уравнение для определения критической температуры, ниже которой происходит образование конденсата. Рассматриваемую модель нетрудно обобщить на случай учета потерь и накачки и вместо (12) решать уравнение

$$
\dot{n}_{k}=P_{k}-D_{k} n_{k}+\sum_{k \neq k^{\prime}}-n_{k}\left(n_{k^{\prime}}+1\right) W_{k k^{\prime}}+n_{k^{\prime}}\left(n_{k}+1\right) W_{k^{\prime}, k},
$$

где два первых слагаемых в правой части уравнения описывают накачку и потери резонаторных мод. Нахождение химического потенциала для ограниченного спектрального интервала проводится в таком случае из условия

$$
\sum_{k} P_{k}-D_{k} n_{k}=0
$$

где $n_{k}$ определяется с помощью (18).

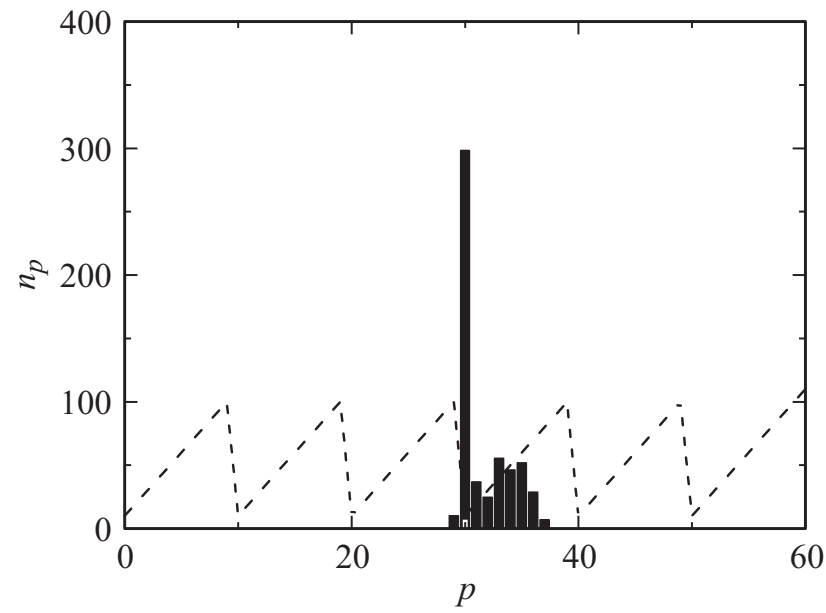

Рис. 1. Установившееся равновесное распределение фотонов в резонаторе. Аргумент $p-$ номер моды резонатора, который мы определили для нашей модели как $p=10 i+j$.

Для иллюстрации процесса установления термодинамического равновесия рассмотрим модель микрорезонатора, близкую к резонатору, использованному в экспериментах $[9,10]$, с высокодобротными высокочастотными продольными модами и низкодобротными низкочастотными поперечными модами. Собственные частоты нашего резонатора представим в виде

$$
\omega_{i j}=i \Omega+j \omega(i=0,1,2, \ldots),(j=0,1,2, \ldots),
$$

заменив индекс $k$ двойным индексом $i j$. Здесь первое слагаемое определяет собственную частоту продольной моды, кратную частоте $\Omega$, второе определяет частоту поперечной моды, кратную частоте $\omega$. Будем предполагать $\Omega \gg \omega$ и поперечные моды двумерными с кратностью вырождения $(j+1)$. Как и выше, каждую моду будем характеризовать коэффициентами накачки и потерь $P_{i j}, D_{i j}$, причем выражая низкую добротность поперечных мод, положим $D_{i j}=(j+1)^{2} D$. Тогда кинетические уравнения (20) можно записать в виде

$$
\begin{aligned}
\dot{n}_{i j} & =P_{i j}-(j+1)^{2} D n_{i j}-\sum_{i^{\prime} j^{\prime} \neq i j} n_{i j}\left(n_{i^{\prime} j^{\prime}}+1\right)\left(j^{\prime}+1\right) \\
& \times W_{i j, i^{\prime} j^{\prime}}+\sum_{i^{\prime} j^{\prime} \neq i j} n_{i^{\prime} j^{\prime}}\left(n_{i j}+1\right)(j+1) W_{i^{\prime} j^{\prime}, i j} .
\end{aligned}
$$

При рассеянии мы будем учитывать взаимодействие только соседних по частотам мод, а вырожденные моды будем считать одинаково заселенными, что обусловливает появление множителей $\left(j^{\prime}+1\right)$ и $(j+1)$ в правой части уравнения.

Мы провели решение системы уравнений (22), выбрав следующие параметры модели:

$$
\begin{gathered}
\omega=1, \quad \Omega=10, \quad T=0.3, \\
i=0,1, \ldots, 5, \quad j=0,1, \ldots, 9, \quad D_{i j}=0.05,
\end{gathered}
$$

единственный отличный от нуля коэффициент накачки $P_{36}=50$. Вероятность перехода между соседними мо- 


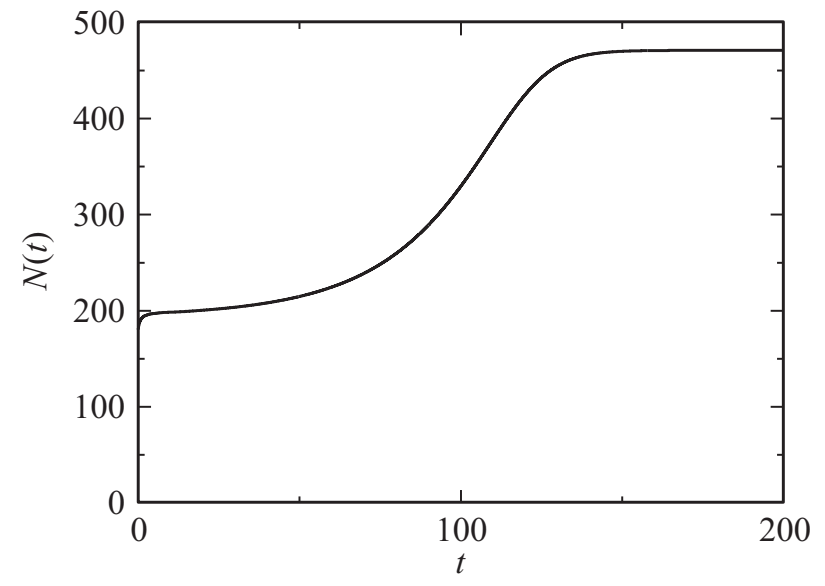

Рис. 2. Динамика установления термодинамического равновесия, $N(t)=\sum_{i, j} n_{i, j}(t)$.

дами будем считать одинаковой для всего спектра резонатора: $w_{k, k^{\prime} \pm 1}=w=10^{-5}$. Резонансную частоту $\omega_{b a}$ мы взяли близкой к частоте накачки, $\omega_{b a}=37.5$. При этом радиационной шириной $\Gamma \ll \omega$ можно пренебречь. Установившееся распределение числа фотонов, полученное в результате решения кинетических уравнений (25), приведено на рис. 1. Пунктиром показано распределение собственных частот резонатора. При выбранных параметрах накачки и затухания мы, действительно, имеем конденсацию фотонов в начале спектрального интервала, соответствующего продольной частоте $3 \Omega$. В общем случае значение частоты конденсации зависит от соотношения величин параметров накачки и добротности. В нашем примере обрывание спектра на частоте $3 \Omega$ обусловлено низкой добротностью высокочастотных поперечных мод резонатора. Динамика установления термодинамического равновесия демонстрируется зависимостью полного числа фотонов в резонаторе от времени (в условных единицах $w^{-1}$ ) на рис. 2 .

\section{Заключение}

Наша работа была инициирована экспериментами [9-11], демонстрирующими эффект конденсации фотонов, который представляет собой аналог БЭК частиц с целым спином и ненулевой массой покоя. Как отмечалось выше, имеется в виду установление квазистационарного термодинамического равновесия на ограниченном спектральном интервале с ненулевой нижней границей, среднее число фотонов в котором поддерживается внешней накачкой. Для оптического диапазона частот даже комнатная температура термостата оказывается достаточной, чтобы обнаружить эффект конденсации: накапливание фотонов на нижней границе спектрального интервала. Этот эффект представляет безусловный интерес, поскольку открывает возможность создания источника монохроматического излучения в результате установления термодинамического равновесия. При учете характеристик микрорезонатора процесс конденсации фотонов может быть описан с помощью решения рассмотренных выше кинетических уравнений.

Выражаю благодарность А.Н. Колерову, обратившему внимание автора на данную проблему, В.А. Малышеву, Н.Н. Розанову и А.С. Трошину за обсуждение.

Работа выполнена при поддержке РФФИ (грант № 15-02-08369-a).

\section{Список литературы}

[1] Einstein A. // Verhandl. Dtsch. Phys. Ges., 1916. V. 18. P. 318 323. (Перевод: Собрание научных трудов. Т. ІІІ. С. 386. М.: Наука, 1966.)

[2] Pauli W. // Zs. Phys. 1923. V. 18. C. 272-286.

[3] Einstein A., Ehrenfest P. // Zs. Phys. 1923. V. 19. P. 301-306.

[4] Компанееи А.С. // ЖЭТФ. 1956. Т. 31. С. 876.

[5] Зельдович Я.Б., Левич Е.В. // ЖЭТФ. 1968. V. 55. Р. 2423 2429.

[6] Einstein A. // Sitzungsber. Preuss. Acad. Wiss., Phys.-math. K1, 3, 1925. (Перевод: Собрание научных трудов. Т. III. С. 489. М.: Наука, 1966.)

[7] Кеттерле В. // УФН. 2003. Т. 173. № 12. С. 1339-1358.

[8] Корнелл Э., Виман К. // УФН. 2003. Т. 173. № 12. С. 1320 1338.

[9] Weitzet M. al. // Neture Phys. 2010. V. 6. P. 512-515.

[10] Weitzet M. al. // Neture. 2010. V. 468. P. 545.

[11] Колеров А.Н., Симунин М.М. // Фотоника. 2013. № 5. Р. 81.

[12] Weisskopf V., Wigner E. // Z. Phys. 1930. V. 63. P. 54; 1930. V. 65. P. 18.

[13] Пойкер К., Трифонов Е.Д. // ФТТ. 1968. Т. 10. С. $1705-$ 1714.

[14] Аветисян Ю.А., Трибонов Е.Д. // УФН. 2015. Т. 185. C. 307-315.

[15] Трифонов Е.Д. // УФН. 2011. Т. 18. С. 747-751.

[16] Загуляев С.Н., Трибонов Е.Д. // УФН. 2010. Т. 180. C. 89-96. 